L3 UPDATE OF THE OPACIN AND OPACIN-NEO TRIALS: 36-MONTHS AND 24-MONTHS RELAPSE-FREE SURVIVAL AFTER (NEO)ADJUVANT IPILIMUMAB PLUS NIVOLUMAB IN MACROSCOPIC STAGE III MELANOMA PATIENTS

${ }^{1} \mathrm{JM}$ Versluis*, 'EA Rozeman, ${ }^{2,3,4} \mathrm{AM}$ Menzies, ${ }^{1}$ ILM Reijers, ${ }^{1} \mathrm{O}$ Krijgsman, ${ }^{1}$ EP Hoefsmit, ${ }^{1} \mathrm{BA}$ van de Wiel, ${ }^{1} \mathrm{~K}$ Sikorska, ${ }^{1} \mathrm{C}$ Bierman, ${ }^{1} \mathrm{P}$ Dimitriadis, ${ }^{2} \mathrm{M}$ Gonzalez, ${ }^{1} \mathrm{~A}$ Broeks, ${ }^{1}$ RM Kerkhoven, ${ }^{2}$ AJ Spillane, ${ }^{1}$ JBAG Haanen, ${ }^{1}$ WJ van Houdt, ${ }^{2,3,5}$ RPM Saw, ${ }^{6} \mathrm{H}$ Eriksson, ${ }^{1} \mathrm{ACJ}$ van Akkooi, ${ }^{2,5} \mathrm{RA}$ Scolyer, ${ }^{1} \mathrm{TN}$ Schumacher, ${ }^{2,4} \mathrm{GV}$ Long, ${ }^{1} \mathrm{CU}$ Blank. ${ }^{1}$ Netherlands Cancer Institute/Antoni van Leeuwenhoek (NKI-AvL), Amsterdam, Netherlands; ${ }^{2}$ Melanoma Institute Australia, Sydney, Australia; ${ }^{3}$ Sydney Medical School, Sydney, Australia; ${ }^{4}$ Royal North Shore Hospital, Sydney, Australia; ${ }^{5}$ Royal Prince Alfred Hospital, Sydney, Australia; ${ }^{6}$ Karolinska Institutet, Stockholm, Sweden

\subsection{6/jitc-2020-ITOC7.3}

Background Before adjuvant checkpoint inhibition the 5-year overall survival (OS) rate was poor $(<50 \%)$ in high-risk stage III melanoma patients. Adjuvant CTLA-4 (ipilimumab, IPI) and PD-1 (nivolumab, NIVO, or pembrolizumab) blockade have been shown to improve relapse-free survival (RFS) and OS (latter only for IPI so far). Due to a broader immune activation neoadjuvant therapy with checkpoint inhibitors might be more effective than adjuvant, as suggested in preclinical experiments. The OpACIN trial compared neoadjuvant versus adjuvant IPI plus NIVO, while the subsequent OpACIN-neo trial tested three different dosing schedules of neoadjuvant IPI plus NIVO without adjuvant therapy. High pathologic response rates of $74-78 \%$ were induced by neoadjuvant IPI plus NIVO. Here, we present the 36- and 24-months RFS of the OpACIN and OpACIN-neo trial, respectively.

Materials and Methods The phase 1b OpACIN trial included 20 stage IIIB/IIIC melanoma patients, which were randomized to receive IPI $3 \mathrm{mg} / \mathrm{kg}$ plus NIVO $1 \mathrm{mg} / \mathrm{kg}$ either adjuvant 4 cycles or split 2 cycles neoadjuvant and 2 adjuvant. In the phase 2 OpACIN-neo trial, 86 patients were randomized to 2 cycles neoadjuvant treatment, either in arm A: $2 \mathrm{x}$ IPI 3 $\mathrm{mg} / \mathrm{kg}$ plus NIVO $1 \mathrm{mg} / \mathrm{kg}$ q3w $(\mathrm{n}=30)$, arm B: $2 \mathrm{x}$ IPI 1 $\mathrm{mg} / \mathrm{kg}$ plus NIVO $3 \mathrm{mg} / \mathrm{kg} \mathrm{q} 3 \mathrm{w}(\mathrm{n}=30)$, or arm C: $2 \mathrm{x}$ IPI 3 $\mathrm{mg} / \mathrm{kg} \mathrm{q} 3 \mathrm{w}$ followed immediately by $2 \mathrm{x}$ NIVO $3 \mathrm{mg} / \mathrm{kg} \mathrm{q} 3 \mathrm{w}$ $(n=26)$. Pathologic response was defined as $<50 \%$ viable tumor cells and in both trials centrally reviewed by a blinded pathologist. RFS rates were estimated using the Kaplan-Meier method.

Results Only 1 of 71 (1.4\%) patients with a pathologic response on neoadjuvant therapy had relapsed, versus 16 of 23 patients (69.6\%) without a pathologic response, after a median follow-up of 36 months for the OpACIN and 24 months for the OpACIN-neo trial. In the OpACIN trial, the estimated 3-year RFS rate for the neoadjuvant arm was $80 \%$ (95\% CI: 59\%-100\%) versus 60\% (95\% CI: 36\%-100\%) for the adjuvant arm. Median RFS was not reached for any of the arms within the OpACIN-neo trial. Estimated 24-months RFS rate was $84 \%$ for all patients (95\% CI: 76\%-92\%); $90 \%$ for arm A (95\% CI: 80\%-100\%), 78\% for arm B (95\% CI: 63\%-96\%) and 83\% for arm C (95\% CI: 70\%-100\%). Baseline interferon- $\gamma$ gene expression score and tumor mutational burden predict response.

Conclusions OpACIN for the first time showed a potential benefit of neoadjuvant IPI plus NIVO versus adjuvant immunotherapy, whereas the OpACIN-neo trial confirmed the high pathologic response rates that can be achieved by neoadjuvant IPI plus NIVO. Both trials show that pathologic response can function as a surrogate markers for RFS.

Clinical trial information NCT02437279, NCT02977052
Disclosure Information J.M. Versluis: None. E.A. Rozeman: None. A.M. Menzies: F. Consultant/Advisory Board; Modest; BMS, MSD, Novartis, Roche, Pierre-Fabre. I.L.M. Reijers: None. O. Krijgsman: B. Research Grant (principal investigator, collaborator or consultant and pending grants as well as grants already received); Modest; BMS. E.P. Hoefsmit: None. B.A. van de Wiel: None. K. Sikorska: None. C. Bierman: None. P. Dimitriadis: None. M. Gonzalez: None. A. Broeks: None. R. M. Kerkhoven: None. A.J. Spillane: None. J.B.A.G. Haanen: B. Research Grant (principal investigator, collaborator or consultant and pending grants as well as grants already received); Modest; BMS, MSD, Neon Therapeutics, Novartis. F. Consultant/Advisory Board; Modest; BMS, MSD, Novartis, Pfizer, AZ/MedImmune, Rocher/Genentech, Ipsen, Bayer, Immunocore, SeattleGenetics, Neon Therapeutics, Celsius Therapeutics, Gadet, GSK. W.J. van Houdt: None. R.P.M. Saw: None. H. Eriksson: None. A.C.J. van Akkooi: B. Research Grant (principal investigator, collaborator or consultant and pending grants as well as grants already received); Modest; Amgen, BMS, Novartis. F. Consultant/Advisory Board; Modest; Amgen, BMS, Novartis, MSD Merck, Merck-Pfizer, 4SC. R.A. Scolyer: F. Consultant/Advisory Board; Modest; MSD, Neracare, Myriad, Novartis. T.N. Schumacher: B. Research Grant (principal investigator, collaborator or consultant and pending grants as well as grants already received); Modest; MSD, BMS, Merck. E. Ownership Interest (stock, stock options, patent or other intellectual property); Modest; AIMM Therapeutics, Allogene Therapeutics, Amgen, Merus, Neogene Therapeutics, Neon Therapeutics. F. Consultant/Advisory Board; Modest; Adaptive Biotechnologies, AIMM Therapeutics, Allogene Therapeutics, Amgen, Merus, Neon Therapeutics, Scenic Biotech. Other; Modest; Third Rock Ventures. G. V. Long: F. Consultant/Advisory Board; Modest; Aduro, Amgen, BMS, Mass-Array, Pierre-Fabre, Novartis, Merck MSD, Roche. C.U. Blank: B. Research Grant (principal investigator, collaborator or consultant and pending grants as well as grants already received); Modest; BMS, Novartis, NanoString. E. Ownership Interest (stock, stock options, patent or other intellectual property); Modest; Uniti Cars, Neon Therapeutics, Forty Seven. F. Consultant/Advisory Board; Modest; BMS, MSD, Roche, Novartis, GSK, AZ, Pfizer, Lilly, GenMab, Pierre-Fabre.

\section{L4 SYNTHETIC AGONISTIC RECEPTOR-ACTIVATING BITES - A MODULAR PLATFORM FOR THE EFFICIENT TARGETING OF ACUTE MYELOID LEUKEMIA}

${ }^{1} \mathrm{M}$ Benmebarek*, ${ }^{1} \mathrm{BL}$ Cadilha, ${ }^{2} \mathrm{M}$ Hermann, ${ }^{1} \mathrm{~S}$ Lesch, ${ }^{2} \mathrm{C}$ Augsburger, ${ }^{2} \mathrm{~B}$ Brauchle, ${ }^{1} \mathrm{~S}$ Stoiber, ${ }^{3} \mathrm{~A}$ Darwich, ${ }^{1} \mathrm{~F}$ Rataj, ${ }^{4} \mathrm{C}$ Klein, ${ }^{2} \mathrm{~K}$ Hopfner, ${ }^{2} \mathrm{M}$ Subklewe, ${ }^{1} \mathrm{~S}$ Endres, ${ }^{1} \mathrm{~S}$ Kobold. ${ }^{1}$ Division of Clinical Pharmacology, Munich, Germany; ${ }^{2}$ Department of Medicine III, Klinikum der Universität München, Munich, Germany; ${ }^{3}$ Mucosal Immunology and Microbiota Unit, Humanitas Research Hospital, Milan, Italy; ${ }^{4}$ Roche Innovation Center Zurich, Schlieren, Switzerland

\subsection{6/jitc-2020-ITOC7.4}

Background Targeted immunotherapies have shown limited success in the context of acute myeloid leukemia (AML). Due to the mutational landscape and heterogeneity attributed to this malignancy and toxicities associated with the targeting of myeloid lineage antigens, it has become apparent that a modular and controllable cell therapy approach with the potential 